\title{
Inseminación artificial a tiempo fijo en vacas con proestro prolongado de 60 y 72 horas $^{1}$
}

\section{Fixed-time artificial insemination in beef cattle with prolonged proestrus of 60 and 72 hours}

\author{
Darwin Omar Yánez-Avalos ${ }^{2}$, Juan Carlos López-Parra ${ }^{2,3}$, Juan Carlos Moyano-Tapia ${ }^{2,3}$, \\ Roberto Orlando Quinteros-Pozo ${ }^{2,3}$, Pablo Roberto Marini ${ }^{2,4}$
}

\begin{abstract}
Resumen
La inseminación artificial a tiempo fijo (IATF) en ganado bovino ha permitido el uso de toros genéticamente superiores para maximizar la calidad de los terneros producidos. Sin embargo, la implementación de esta técnica reproductiva dificulta su aplicación en hembras bovinas que están con cría al pie durante varios meses de lactancia. Una solución inmediata a la disminución de la fertilidad en vacas incluye la utilización de hormonas. Se ha desarrollado una serie de tratamientos hormonales que es capaz de controlar el momento de la primera inseminación artificial (IA) y las inseminaciones posteriores en vacas no preñadas y para el tratamiento de vacas en anestro. El objetivo de este trabajo fue evaluar dos protocolos con proestro prolongado con inseminación artificial a tiempo fijo (IATF) a las 60 o 72 horas, en vacas doble propósito de la Amazonía Ecuatoriana. El trabajo se realizó desde octubre de 2015 a octubre de 2016. Se inseminaron 226 vacas multíparas cruzas Pardo Suizo con cría al pie, a las 60 (T1, $\mathrm{n}=115)$ y $72(\mathrm{~T} 2, \mathrm{n}=111)$ horas de retirado el dispositivo con progesterona $(0,5 \mathrm{~g})$. La tasa de concepción para $\mathrm{T} 1$ fue del $61 \%(70 / 115)$ y para T2: el $47 \%$ de preñez $(52 / 111)$, con diferencias significativas $(\mathrm{p} \leq 0,05)$. El celo en promedio fue de $70,8 \%$, sin diferencia significativa entre tratamientos $(\mathrm{P}=3,15 ; \mathrm{p} \geq 0,082)$, para $\mathrm{T} 1$ fue del $76 \%$ y para $\mathrm{T} 2$ del $65 \%$. El crecimiento folicular al retiro del dispositivo con progesterona (T1: 9,53 $\pm 0,11 \mathrm{~mm}$; T2: 9,87 $\pm 0,12 \mathrm{~mm}$ ), la IATF (T1: $12,3 \pm 0,12 \mathrm{~mm}$; T2: 11,9 $\pm 0,12 \mathrm{~mm}$ ) y el tamaño del cuerpo lúteo (T1: $23,1 \pm 0,14 \mathrm{~mm}$; T2: 22,8 0,15 $\mathrm{mm})$ ), no mostraron diferencias significativas entre ambos protocolos $(\mathrm{p} \geq 0,05)$. Ambos tratamientos no mostraron diferencias significativas para el desarrollo folicular al momento del retiro del dispositivo de IATF y en el tamaño del cuerpo lúteo medido al día siete posterior a la misma. La tasa de preñez fue superior para el protocolo de 60 horas.
\end{abstract}

Palabras clave: estradiol, progesterona, folículos ováricos, ovulación inducida, gestación.

\begin{abstract}
Fixed- time artificial insemination (IATF, in Spanish) in beef cattle has provided the use of genetically superior bulls in order to maximize the quality of calves. However, implementing this reproductive technique has proved to be difficult to apply in heifers nursing calves for several months of lactancy. An immediate solution to fertility decline

1 Recibido: 3 de julio, 2017. Aceptado: 10 de agosto, 2017. Este trabajo formó parte del trabajo final de la Especialización en Reproducción Bovina del primer autor. Instituto de Reproducción Animal y Universidad Nacional de Córdoba, Argentina.

2 Centro Latinoamericano de Estudios de Problemáticas Lecheras (CLEPL). Ovidio Lagos y Ruta 33 CP (2170) Casilda Provincia de Santa Fe, Argentina.dr.daviyanez@gmail.com

3 Universidad Estatal Amazónica-Centro de Investigación, Posgrado y Conservación Amazónica, Ecuador. Paso Lateral Km2 1/2 Vía Napo Puyo, Pastaza, Ecuador. reprogenetics.jclopez@gmail.com; juancamt@hotmail.com; sverqp@hotmail.com

4 Universidad Nacional de Rosario (UNR), Facultad de Ciencias Veterinarias y Carrera de Investigador Científico (CIC), Argentina. Ovidio Lagos y Ruta 33 CP (2170) Casilda Provincia de Santa Fe. pmarini@unr.edu.ar
\end{abstract}


of cattle includes the use of hormones. Some hormonal treatments have been developed which are able to control the moment of the first artificial insemination (IA) and the following inseminations in non- pregnant heifers as well as for the treatment of anestrus. The objective of this work was to evaluate two protocols with prolonged proestrus performing artificial insemination at fixed time at 60 or 72 hours in dual purpose cattle from the Ecuadorian Amazon. The study was held from October 2015 to October 2016. A total of 226 multiparious Brows Swiss cattle with nursing calves were inseminated at $60(\mathrm{~T} 1, \mathrm{n}=115)$ and at $72(\mathrm{~T} 2, \mathrm{n}=111)$ hours after removing the progesterone device., The conception rate for T1 was 61\% (70/115) and for T2: 47\% of pregnancy (52/111), showing significant differences $(\mathrm{p} \leq 0.05)$. The estrus was expressed on average by $70.8 \%$, with no significant difference between the protocols $(\mathrm{P}$ $=3.15, \mathrm{p} \geq 0.082)$, for $\mathrm{T} 1$ it was $76 \%(87 / 115)$ and for $\mathrm{T} 2$ of $65 \%$. The follicular growth at the withdrawal of the progesterone device $(\mathrm{T} 1: 9.53 \pm 0.11 \mathrm{~mm}, \mathrm{~T} 2: 9.87 \pm 0.12 \mathrm{~mm})$, the IATF $(\mathrm{T} 1: 12.3 \pm 0.12 \mathrm{~mm}$; T2: $11.9 \pm 0.12$ $\mathrm{mm}$ ), and the corpus luteum size (T1: $23.10 .14 \pm \mathrm{mm}$; T2:22.8 $0.15 \pm \mathrm{mm}$ ) did not show significant differences $\mathrm{p} \geq 0.5$ between both protocols. It was concluded that both protocols did not show significant differences regarding follicular development at the time of withdrawal of the IATF device and the size of the corpus luteum size measured at day seven after withdrawal. However, the pregnancy rate was higher for the 60-hour protocol.

Keywords: estradiol, progesterone, ovarian follicles, induced ovulation, pregnancy.

\section{Introducción}

La inseminación artificial a tiempo fijo (IATF) en ganado bovino ha permitido el uso de toros genéticamente superiores para maximizar la calidad de los terneros producidos. Sin embargo, la implementación de esta técnica reproductiva dificulta su aplicación en hembras bovinas que están con cría al pie durante varios meses de lactancia. Una solución inmediata a la disminución de la fertilidad en vacas incluye la utilización de hormonas. Se ha desarrollado una serie de tratamientos hormonales capaces de controlar el momento de la primera inseminación artificial (IA) y las inseminaciones posteriores en vacas no preñadas, y para el tratamiento de vacas en anestro (Cutaia, 2011).

El uso de la inseminación artificial ha permitido obtener excelentes resultados, ya que por medio de diversos protocolos se ha logrado acortar los días abiertos en las vacas, con varias ventajas en la implementación de programas de inseminación a tiempo fijo (IATF), dentro de las más destacadas están la mejora genética de la progenie y, de capital importancia, la facilidad de partos evitando las distocias (Cutaia et al., 2003). Así, la eficiente multiplicación de animales superiores, proporciona un mayor retorno económico a la actividad (Baruselli et al., 2005).

Existen actualmente en el mercado, dispositivos eficientes que liberan progesterona y que son mantenidos en la vagina por un período de siete u ocho días (Bó et al., 2001). El tratamiento que se utiliza consiste en administrar 2 $\mathrm{mg}$ de benzoato de estradiol por vía intramuscular junto con la inserción del dispositivo intravaginal con liberación de progesterona en lo que se denomina el día 0 del tratamiento. En el día siete u ocho se extrae el dispositivo y se aplica una dosis de prostaglandina luteolítica $\left(\mathrm{PGF}_{2 \mathrm{a}}\right)$ y $1 \mathrm{mg}$ de benzoato de estradiol (BE) (IM) 24 h más tarde (Bó y Baruselli, 2002), o 0,5 mg de cipionato de estradiol (ECP) en el mismo momento de la remoción del dispositivo.

Durante la última década, se han desarrollado nuevas generaciones de protocolos denominados "tratamientos cortos", que han demostrado mejorar la taza de preñez, y se fundamentan en que disminuyen el período de inserción de dispositivos con progesterona, reduce en el período de dominancia del folículo y prolongan el proestro previo a la ovulación (Bridges et al., 2008).

Desde el año 2011 se viene realizando una serie de experimentos, con el objetivo de desarrollar nuevos tratamientos usando BE y progesterona para sincronizar el inicio de una nueva onda folicular con un periodo reducido de inserción de dispositivo con progesterona de seis días en lugar de siete u ocho días, y la administración de GnRH como inductor de ovulación a las 72 h, desde la remoción del dispositivo junto con la IATF para desarrollar un proestro prolongado. A este tratamiento se ha denominado J-Synch (de-la-Mata y Bó, 2012), en el cual se obtuvieron proestros con promedios de 95 y 97 h, lo que hace suponer que mejoraría las tasas de 
gestación (de-la-Mata et al., 2015), ya que, se demostró que la calidad embrionaria puede verse afectada cuando la dominancia de un folículo ovulatorio aumenta más de 1,5 días (Cerri et al., 2009) y la prolongación del proestro se correlaciona con mayores concentraciones séricas de estradiol, aumentando así la fertilidad en la IATF (Bridges et al., 2008; 2010).

El objetivo de este trabajo fue evaluar dos protocolos con proestro prolongado e inseminación artificial a tiempo fijo a las 60 o 72 horas, en vacas doble propósito de la Amazonía Ecuatoriana.

\section{Materiales y métodos}

Esta investigación se realizó en la povincia de Pastaza, Ecuador, desde octubre del 2015 a octubre del 2016. La provincia de Pastaza es una zona con una precipitación fluvial de 4000 - $5000 \mathrm{~mm} / \mathrm{año}$, el clima es cálido y húmedo, con una temperatura que varía entre los 18 y $24^{\circ} \mathrm{C}$. Además, cuenta con una topografía irregular, fincas con un área promedio de 50 ha, conformadas por pastizales propios de la zona (Gramalote) Axonopusscoparius, y pequeñas parcelas agrícolas.

Se utilizaron 226 vacas doble propósito que se encontraban amamantando, cruzas de Pardo Suizo x Bos indicus de 34 a 65 meses de edad, con un peso de entre 350 y $380 \mathrm{~kg}$ y una condición corporal de 2,5 a 3 (Ben et al., 2002), las mismas se encontraban pastoreando en campo natural. Para esta investigación se emplearon dos grupos de vacas en forma aleatoria.

\section{Tratamientos}

En el tratamiento 1 (J-Synch 60 horas IA +eCG) (n=115), en el día cero se realizó la primera evaluación ecográfica, con el propósito de analizar el estatus uterino y ovárico, descartando posibles afecciones reproductivas y sobre todo animales acíclicos, se aplicó un dispositivo con progesterona $(0,5 \mathrm{~g})$ y $2 \mathrm{mg}$ de benzoato de estradiol vía intramuscular. En el día seis se removió el dispositivo con progesterona y se observó la calidad del implante (presencia o ausencia de vaginitis), se administró $500 \mu \mathrm{g}$ de cloprostenol más 500 UI de gonadotropina sérica de yegua preñada (eCG - Folligon Huixquilucan-Estado de México), y se realizó una medición ecográfica del desarrollo folicular y seguimiento visual de la presencia de celo (moco vaginal y en flancos) anticipada a la inseminación artificial a tiempo fijo. Al día nueve o proestro a las 60 h de la aplicación de prostaglandina luteolítica (PGF), se realizó otra medición con ecografía para ver el comportamiento de los folículos con un proestro prolongado y se aplicó 2,5 $\mathrm{ml}$ de acetato de buserelina $(\mathrm{GnRH})$ vía intramuscular; las vacas se inseminaron a las $60 \mathrm{~h}$ de retirado el dispositivo con progesterona.

Tratamiento 2 (J-Synch 72 horas IA + eCG) (n=111), el día cero se realizó la primera evaluación ecográfica, con el propósito de analizar el estatus uterino y ovárico, se descartó posibles afecciones reproductivas, sobre todo animales acíclicos, y se aplicó un dispositivo con progesterona $(0,5 \mathrm{~g})$ y $2 \mathrm{mg}$ de benzoato de estradiol vía intramuscular. En el día seis se removió el dispositivo con progesterona, revisando la calidad del implante de progesterona (presencia o ausencia de vaginitis), y se administró $500 \mu \mathrm{g}$ de cloprostenol más 500 UI de gonadotropina sérica de yegua preñada; se realizó una medición ecográfica del desarrollo folicular y seguimiento visual de la presencia de celo (moco vaginal y en flancos) anticipada a la inseminación artificial a tiempo fijo. Al día nueve o proestro a las 72 h de la aplicación de PGF, se realizó otra medición con ecografía para ver el comportamiento de los folículos con un proestro prolongado y se aplicó $2,5 \mathrm{ml}$ de acetato de buserelina $(\mathrm{GnRH})$ vía intramuscular; las vacas se inseminaron a las $72 \mathrm{~h}$ de retirado el dispositivo con progesterona.

Para la inseminación artificial a tiempo fijo (IATF) se utilizó semen congelado/descongelado (Bernardi et al., 2011) de un solo toro de fertilidad probada, aunque igualmente antes de utilizarlo se analizó. Los animales 
se inseminaron por un mismo técnico. La distribución de los tratamientos fue completamente al azar, con cuatro repeticiones (T1: 28, 29, 29 y 29 y T2: 27, 28, 28 y 28, vacas con cada uno de los tratamientos). Los celos se observaron por medio de decoloración de pintura de la base de la cola y moco vaginal en momentos previos a la ecografía e IATF, la detección se realizó 24 h después del retiro del dispositivo, una vez por la mañana y una vez por la tarde (de los días 7, 8 y 9).

La evaluación de la calidad del dispositivo se realizó al momento de retirarlo del tracto vaginal, y se determinó la presencia y calidad de moco: limpio (moco cristalino) o sucio (moco turbio purulento). La evaluación del desarrollo folicular se realizó con ultrasonografía, al día cero en la implantación del dispositivo intravaginal bovino de progesterona, el día seis al retiro del dispositivo intravaginal bovino de progesterona y al momento de la IATF (60 y $72 \mathrm{~h}$ de retirado el dispositivo intravaginal bovino de progesterona), con la finalidad de realizar un seguimiento a la dinámica ovárica. También, al día siete post inseminación, se midió a través de ecografía el tamaño de cuerpo lúteo, para ello, siempre se tomaron dos medidas (alto - ancho en mm) de cada estructura (cuerpo lúteo o folículo) y se promedió entre ambas para obtener una medida más aproximada. El diagnóstico de gestación se determinó por medio de ultrasonografía entre los 40 y 45 días posterior a la IATF (I Bex-Pro versión 2011, transductor L 6.2 pero es de $5 \mathrm{MHz}$ lineal).

\section{Variables analizadas}

En la realización de este estudio se analizaron las siguientes variables:

- Detección de celos en porcentaje (\%).

- Vaginitis: presencia o ausencia, al retiro del implante de progesterona.

- Desarrollo folicular en mm (DF).

- Tamaño de cuerpo lúteo post ovulación en mm (TCL).

- Tasa de preñez (TP) en porcentaje (\%).

- Días abiertos (fecha de tratamiento - fecha de parto de cada vaca) en días.

\section{Análisis estadístico}

Para cada variable estudiada se estimaron la media aritmética (X) y el error estándar (EE). Se probó si existían diferencias significativas entre los tratamientos, mediante la aplicación de análisis de variancia (ANOVA) a un criterio de clasificación y pruebas de comparaciones múltiples de Tukey-Kramer HSD ( $\leq 00,05)$. La relación entre días de paridas y la proporción del dispositivo intravaginal limpio o sucio, se evaluó con una prueba de homogeneidad basada en la estadística Chi-cuadrado $(\mathrm{P}<0,05)$ y prueba de hipótesis para variables continuas, según t-Student al $\mathrm{P}<0,05$. Los análisis estadísticos se realizaron con base en el programa JMP en su versión 5.0 para Windows.

\section{Resultados}

\section{Tasa de preñez}

La tasa de preñez de los protocolos T1 (60 horas IA +eCG) y T2 (72 horas IA + eCG), mostraron diferencia significativa $(\mathrm{P} \leq 0,05)$. Se obtuvo un 61\% (70/115) para el primer tratamiento y 47\% (52/111) para T2. 


\section{Expresión de celo}

El celo se expresó en promedio en un $70,8 \%$, no hubo diferencia significativa entre los protocolos $(\mathrm{p}=3,15$; $\mathrm{p} \geq 0,082$ ), se obtuvo un 75,7\% (87/115) para el $\mathrm{T} 1$, y un $64,9 \%$ para $\mathrm{T} 2$ (Cuadro 1 ).

Cuadro 1. Cantidad de vacas cruzas Pardo Suizo en celo y sin celo, según tratamientos hormonales para los protocolos con proestro prolongado J-Synch 60h y J-Synch 72h. Pastaza. Ecuador. 2015-2016.

Table 1. Number of crosses Brown Swiss cattle in estrus and without estrus according to hormonal treatments for protocols with prolonged proestrus J-Synch 60h and J-Synch 72h. Pastaza, Ecuador. 2015-2016.

\begin{tabular}{ccccc}
\hline Tratamiento & Animales & Celo & Sin Celo & TOTAL \\
\hline J- Synch $60 \mathrm{~h}$ & 115 & 87 & 28 & 75,7 \\
J- Synch 72 h & 111 & 72 & 39 & 65,9 \\
Total & 226 & 159 & 67 & 70,8 \\
\hline
\end{tabular}

De las vacas que manifestaron celo, la tasa de preñez para T1 fue del 62\% (54/87) y del T2 fue del 50\% (36/72), los cuales no mostraron diferencia significativa, al igual que las vacas $\sin$ presencia de celo, que fueron $57,1 \%$ $(16 / 28)$ y $41 \%(16 / 39)(\mathrm{p}=0,401, \mathrm{p} \geq 0,526)$ para $\mathrm{T} 1$ y $\mathrm{T} 2$, respectivamente (Cuadro 2$)$.

Cuadro 2. Proporción y porcentaje de celo y preñez en vacas cruzas Pardo Suizo según tratamiento hormonal para los protocolos con proestro prolongado J-Synch 60h y J-Synch 72h. Pastaza. Ecuador. 2015-2016.

Table 2. Proportion and percentage of estrus and pregnancy of crosses Brown Swiss cattle according to hormonal treatment for protocols with prolonged proestrus J-Synch 60h and J-Synch 72h. Pastaza, Ecuador. 2015-2016.

\begin{tabular}{lcccccc}
\hline Tratamiento & Celo & \% Preñez & Sin Celo & \% Preñez & Total & \% Preñez \\
\hline J-Synch $60 \mathrm{~h}$ & $54 / 87$ & 62 & $16 / 28$ & 57,1 & $70 / 115$ & 61 \\
J-Synch 72 h & $36 / 72$ & 50 & $16 / 39$ & 41 & $52 / 111$ & 47 \\
\hline
\end{tabular}

\section{Presencia o ausencia de vaginitis al retiro del dispositivo con progesterona}

Respecto a la presencia o ausencia de vaginitis al retiro del dispositivo de progesterona, no existió diferencia significativa entre los dos tratamientos $(\mathrm{p}>0,05)$ (Figura 1$)$.

Se agruparon a las vacas según los días de paridas (90 - 120 y 150 días), en donde se observó un mayor porcentaje de vaginitis en las vacas de 90 días post parto, en comparación con las vacas con un período abierto de 120 días, siendo estas últimas las que mejor se comportaron en este tratamiento (Figura 1). Las vacas con intervalo de días abiertos (120 días), inseminadas a las $60 \mathrm{~h}$ de retirado el dispositivo, tuvieron mayor posibilidad de preñez, sin importar la calidad del dispositivo de progesterona al retiro (presencia o ausencia de vaginitis), con un 69\% (24/35) (Cuadro 3).

Al agrupar las vacas, a los 60,90,120 y 150 días de paridas e inseminadas a las $72 \mathrm{~h}$ de retirado el dispositivo, se observó que, en vacas de 60 días post parto, existió mayor proporción de dispositivos de progesterona con presencia de vaginitis, en comparación con las vacas con período abierto mayor (Figura 2). 


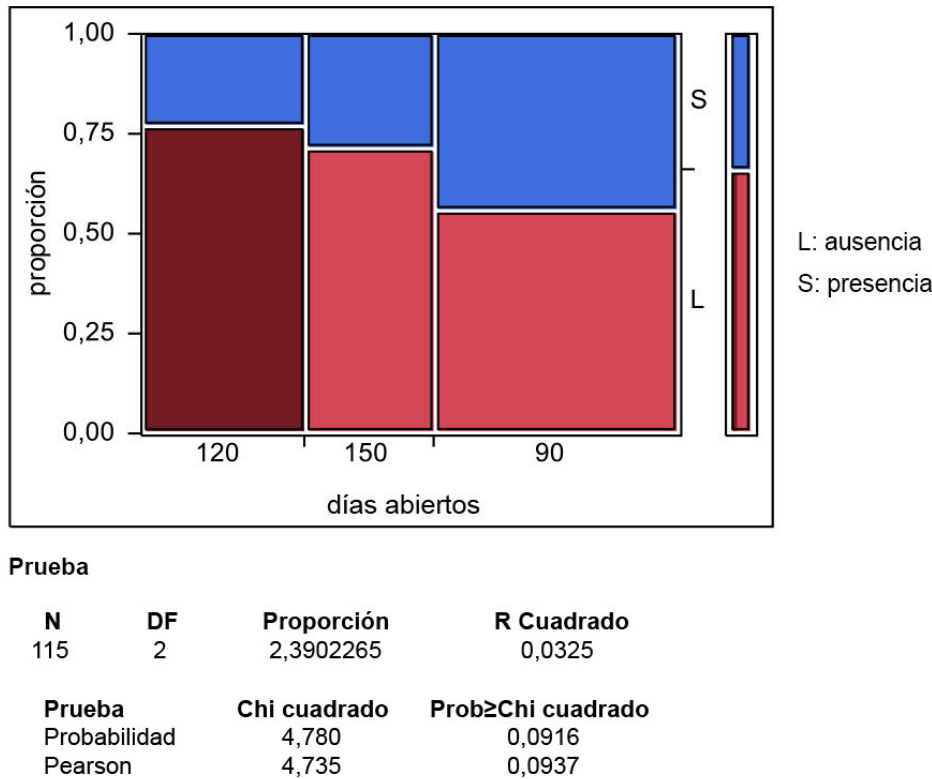

Figura 1. Proporción de vaginitis al retiro del dispositivo con progesterona, utilizado en vacas multíparas cruzas Pardo Suizo, según días abiertos para el protocolo con proestro prolongado J-Synch-60h. Pastaza, Ecuador. 2015-2016.

Figure 1. Proportion of presence or absence of vaginitis at the withdrawal of the progesterone device in multiparous Brown Swiss cattle according to days open for prolonged proestrus protocol J-Synch-60h. Pastaza, Ecuador. 2015-2016.

\section{Dinámica folicular al retiro del dispositivo, y momento de la IATF}

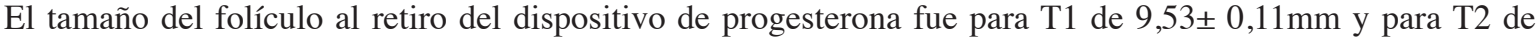

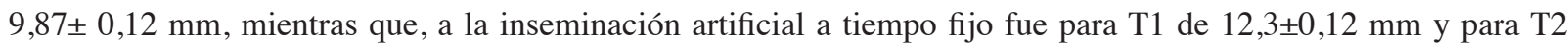
de $11,9 \pm 0,12 \mathrm{~mm}$, sin mostrar diferencias significativas en el tamaño del folículo ambos protocolos y momentos.

Para el J-Synch 60 h, se observó que las vacas que tuvieron 120 días de paridas al momento del tratamiento, mostraron, con diferencias significativas $(\mathrm{p} \leq 0,05)$, un mayor diámetro del cuerpo lúteo al retiro del dispositivo de progesterona $(10,0 \pm 0,19 \mathrm{~mm})$ y a la inseminación artificial a tiempo fijo $(12,8 \pm 0,24 \mathrm{~mm})$ (Cuadro 4).

\section{Tamaño del cuerpo lúteo a los siete días post inseminación}

El diámetro del cuerpo lúteo medido al día 7 , no difirió significativamente entre tratamientos $(\mathrm{p}>0,05)(\mathrm{T} 1$ :

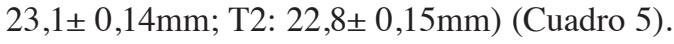

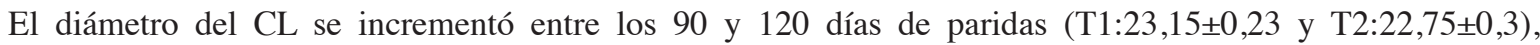
existiendo más posibilidades de preñez. 
Cuadro 3. Presencia o ausencia de vaginitis luego de retirar el dispositivo con progesterona por tratamiento con los protocolos con proestro prolongado J-Synch 60h y J-Synch 72h en vacas multíparas cruzas Pardo Suizo. Pastaza, Ecuador. 2015-2016.

Table 3. Presence or absence of vaginitis after withdrawal of the progesterone device acoording to treatment for protocols with prolonged proestrus J-Synch 60h and J-Synch 72h in multiparious Brown Swiss cattle. Pastaza, Ecuador. 2015-2016.

\begin{tabular}{|c|c|c|c|c|c|c|}
\hline \multicolumn{7}{|c|}{ Grupo de vacas con 90 díasabiertos a la IATF } \\
\hline Tratamiento & Ausencia & \% Preñez & Presencia & \% Preñez & Total & $\%$ Preñez \\
\hline J-Synch 60h & $14 / 25$ & 56 & $12 / 20$ & 60 & $26 / 45$ & 57,8 \\
\hline J-Synch $72 \mathrm{~h}$ & $12 / 23$ & 52,2 & $5 / 12$ & 41,7 & $17 / 35$ & 48,6 \\
\hline Total & & & & & & 53,1 \\
\hline \multicolumn{7}{|c|}{ Grupo de vacas con 120 días abiertos a la IATF } \\
\hline Tratamiento & Ausencia & \% Preñez & Presencia & \% Preñez & Total & \% Preñez \\
\hline J-Synch 60h & $18 / 27$ & 66,6 & $6 / 8$ & 75 & $24 / 35$ & 68,6 \\
\hline J-Synch $72 \mathrm{~h}$ & $8 / 18$ & 44,4 & $5 / 9$ & 55,6 & $13 / 27$ & 48,1 \\
\hline Total & & & & & & 58,3 \\
\hline \multicolumn{7}{|c|}{ Grupo de vacas con 150 días abiertos a la IATF } \\
\hline Tratamiento & Ausencia & \% Preñez & Presencia & \% Preñez & Total & \% Preñez \\
\hline J-Synch 60h & $12 / 20$ & 60 & $5 / 8$ & 62,5 & $17 / 28$ & 60,7 \\
\hline J-Synch $72 \mathrm{~h}$ & $8 / 17$ & 47,1 & $1 / 2$ & 50,0 & $9 / 19$ & 47,4 \\
\hline Total & & & & & & 54 \\
\hline
\end{tabular}
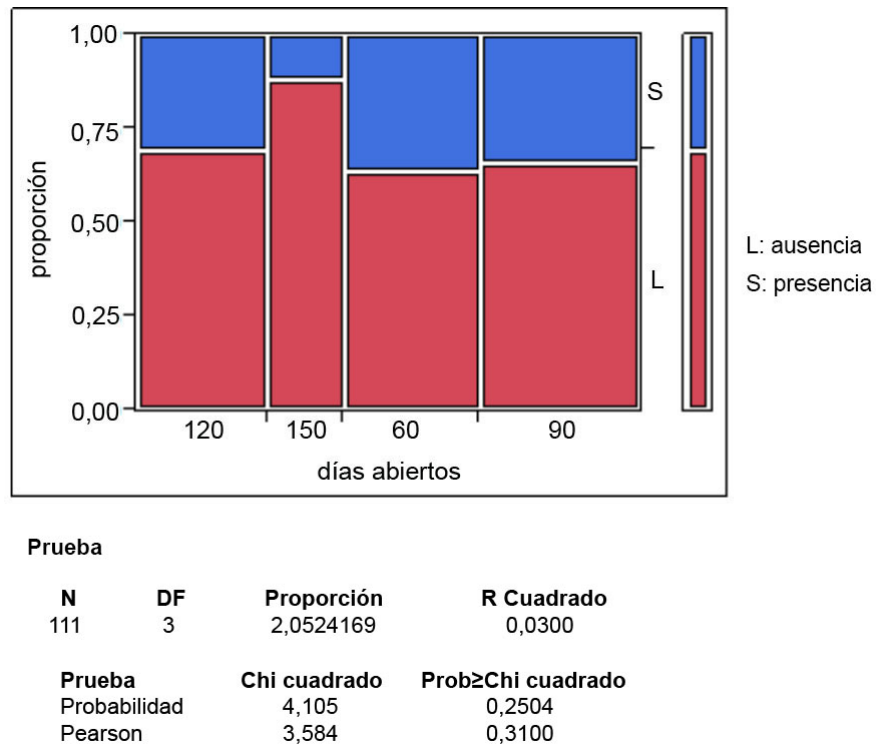

Figura 2. Proporción de vaginitis al retiro del dispositivo con progesterona, utilizado en vacas multíparas cruzas Pardo Suizo, según días abiertos para el tratamiento con el protocolo con proestro prolongado JSinch-72. Pastaza, Ecuador. 2015-2016.

Figure 2. Proportion of presence or absence of vaginitis at the withdrawal of the progesterone device in multiparous Brown Swiss cattle according to days open for prolonged proestrus protocol JSinch-72. Pastaza, Ecuador. 2015-2016. 
Cuadro 4. Promedios y errores estándar de la dinámica folicular $(\mathrm{mm})$ en los protocolos con proestro prolongado J-Synch $60 \mathrm{~h}$ y J-Synch 72h, utilizados en vacas multíparas cruzas Pardo Suizo. Pastaza, Ecuador. 2015-2016.

Table 4. Mean and standard errors of follicular dynamics $(\mathrm{mm})$ in both protocols with prolonged proestrus J-Synch $60 \mathrm{~h}$ and J-Synch 72h in multiparous Brown Swiss cattle. Pastaza, Ecuador. 2015-2016.

\begin{tabular}{ccccc}
\hline & \multicolumn{2}{c}{ J-Synch 60 h } & \multicolumn{2}{c}{ J-Synch 72 h } \\
\hline P.A./días & Retiro de DIB & IATF & Retiro de DIB & IATF \\
\hline 60 & & & $9,3 \pm 0,2 \mathrm{a}$ & $11,4 \pm 0,22 \mathrm{~b}$ \\
90 & $9,1 \pm 0,19 \mathrm{~b}$ & $12,0 \pm 0,17 \mathrm{~b}$ & $10,0 \pm 0,2 \mathrm{a}$ & $12,4 \pm 0,19 \mathrm{a}$ \\
120 & $10,0 \pm 0,19 \mathrm{a}$ & $12,8 \pm 0,24 \mathrm{a}$ & $10,0 \pm 0,2 \mathrm{a}$ & $11,7 \pm 0,2 \mathrm{ab}$ \\
150 & $9,6 \pm 0,22 \mathrm{ab}$ & $12,2 \pm 0,22 \mathrm{ab}$ & $10,2 \pm 0,3 \mathrm{a}$ & $12,1 \pm 0,3 \mathrm{ab}$ \\
\hline
\end{tabular}

Cuadro 5. Promedios y errores estándar del tamaño en mm del cuerpo lúteo a los siete días post inseminación artificial en los protocolos con proestro prolongado J-Synch $60 \mathrm{~h}$ y J-Synch $72 \mathrm{~h}$, utilizados en vacas multíparas cruzas Pardo Suizo. Pastaza. Ecuador. 2015-2016.

Table 5. Mean and standard errors of size in $\mathrm{mm}$ of the corpus luteum at seven dayspost insemination in both protocols with prolonged proestrus J-Synch 60h and J-Synch 72h in multiparous Brown Swiss cattle. Pastaza. Ecuador. 2015-2016.

\begin{tabular}{ccc}
\hline P.A./días & J-Synch $\mathbf{6 0} \mathbf{h}$ & J-Synch 72 h \\
\hline 60 & & $22,6 \pm 0,3 \mathrm{a}$ \\
90 & $22,7 \pm 0,23 \mathrm{~b}$ & $23,0 \pm 0,2 \mathrm{a}$ \\
120 & $23,6 \pm 0,23 \mathrm{a}$ & $22,5 \pm 0,3 \mathrm{a}$ \\
150 & $23,2 \pm 0,27 \mathrm{ab}$ & $23,1 \pm 0,4 \mathrm{a}$ \\
\hline
\end{tabular}

\section{Discusión}

Las vacas del tratamiento 1 (60 horas IA +eCG) tuvieron mayor duración de proestro en comparación con las vacas del tratamiento 2 ( 72 horas IA $+\mathrm{eCG}$ ), con una diferencia entre tratamientos de aproximadamente $12 \mathrm{~h}$. Aunque las concentraciones de estradiol no se midieron en este trabajo, este resultado podría ser explicado en parte, porque la prolongación del proestro generó mayores concentraciones séricas circulantes de estradiol, producido por el folículo dominante, lo que favoreció la madurez folicular y mejoró la fertilidad (Bridges et al., 2008; 2010; 2012; 2014).

El uso de un protocolo con proestro prolongado (J-Synch), mejoró la tasa de preñez $(\mathrm{p} \leq 0,05)$ en vacas doble propósito de la Amazonia Ecuatoriana, cuando se inseminó a las 60 h (61\%) de retirado el dispositivo con progesterona, en comparación con la inseminación a las 72 h (47\%). Menchaca et al. (2015) señalaron que, el tratamiento J-Synch al recibir eCG al momento de la IATF el día noveno en la mañana y la tarde (60 y $72 \mathrm{~h}$ después de retirar el DIB), a las $60 \mathrm{~h}$, tuvo el 54,4\% de preñez (131/241) y a las $72 \mathrm{~h}$ tuvo el 53,0\% (131/247), con una tendencia a mejorar. También, de-la-Mata et al. (2015) mostraron que, la adición de eCG al protocolo J-Synch proporcionó una ventana más amplia de tiempos de inseminación, y además, facilitó la IATF en grandes grupos de vaquillas de carne, en donde el grupo J-Synch inseminadas a las $60 \mathrm{~h}$, tuvieron tasas de preñez mayores, de 60,6\% $(157 / 259)(\mathrm{p}<0,05)$, que aquellas del mismo grupo que fueron IATF a las $72 \mathrm{~h}$.

En cuanto a la expresión de celo en los tratamientos, no se observó diferencia significativa, coincidiendo con de-la-Mata et al. (2015), donde la tasa de detección de celo no difirió entre grupos en protocolos J-Synch. Sin 
embargo, Pitaluga et al. (2013), encontraron mayor proporción de vacas en estro, una mejor respuesta ovulatoria y una tendencia a mejorar el diámetro de cuerpo lúteo durante la fase luteal temprana en vacas en las cuales se manipuló el período de proestro con gonadotrofinas (eCG) y estradiol (CPE).

La dinámica folicular medida al retiro del dispositivo con progesterona y a la IATF, no tuvo diferencia significativa $(p>0,05)$, estos resultados coinciden con varios autores como Núñez-Olivera et al. (2014), quienes en su trabajo analizaron el efecto de la prolongación del proestro sobre el folículo ovulatorio, asociado con una dosis de eCG y GnRH a las 48 y $72 \mathrm{~h}$ de retirar los dispositivos de progesterona en vaquillas de carne, obtuvieron un diámetro folicular $(\mathrm{mm})$ a las $48 \mathrm{~h}$ de retiro del dispositivo de $8,8 \pm 0,4$ y a la IATF de 12,3 $\pm 0,5$, mientras que, a las $72 \mathrm{~h}$ de retiro del dispositivo fue de $8,2 \pm 0,3$ y a la IATF de $13,5 \pm 0,3$, sin encontrar diferencias significativas.

Se ha reportado que vacas con folículos más grandes están más cerca de la ovulación (Van-Eerdenburg et al., 2002). Esto coincide con lo encontrado por Yánez et al. (2016), en donde las vacas que tuvieron un mayor desarrollo folicular a la inseminación, tuvieron una posibilidad catorce veces más de quedar preñadas (preñadas: 13,4 $\pm 1,1 \mathrm{~mm}$; vacías: $10,4 \pm 1,3 \mathrm{~mm})$.

La capacidad ovulatoria de un folículo dominante $(>10 \mathrm{~mm})$ se adquiere luego que este supera el diámetro de desviación (>8,5 mm), tal como fue reportado en un experimento con vacas lecheras (Sartori et al., 2001). En vaquillas de carne se encontró un máximo de probabilidad de preñez cuando el tamaño folicular en el momento de la IA fue de 12,8 mm, mientras que, al presentar folículo dominante por debajo de 10,7 mm o por encima de 15,7 $\mathrm{mm}$, tuvieron menos oportunidades de lograr una preñez (Perry et al., 2007).

Hubo un incremento del diámetro de desarrollo folicular al momento de retirar el dispositivo de progesterona y a la IATF, con una tasa de preñez creciente en vacas desde los 90 a 120 días post parto, sometidas a ambos tratamientos estudiados, lo cual pudo deberse al tiempo necesario para que el animal mejore su condición, ya que el anestro post parto, con el consiguiente incremento del intervalo entre partos, es una de las principales causas de pérdidas económicas de la ganadería bovina bajo condiciones tropicales (Hansel y Alila, 1984), influenciado por el consumo y calidad del alimento, variaciones en el peso, condición corporal al parto e interacciones entre estos factores.

Uno de los principales problemas en los rodeos es el retraso en el reinicio de la actividad ovárica después del parto, Guáqueta et al. (2014). Motta-Delgado et al. (2012) encontraron valores de 452 \pm 100 días de intervalo partoparto en vacas de doble propósito, basado en el mestizaje de razas cebuínas con Holstein. En las condiciones de la Amazonía Ecuatoriana, los valores del intervalo parto-concepción estuvieron entre los 90 y 150 días, lo que daría un intervalo parto-parto futuro de 82 y 22 días por debajo de lo citado anteriormente.

No se observó diferencia significativa en el tamaño del cuerpo lúteo al día 7 post inseminación, pero sí en el incremento del diámetro de CL en vacas con 120 días abiertos, existiendo mayores posibilidades de preñez. En un estudio se mostró que, el desarrollo luteal entre los días 4 y 13 luego de la ovulación, el volumen del cuerpo lúteo tendió a ser mayor ( $\mathrm{p}=0,074)$ en vaquillas del grupo J-Synch en comparación al convencional (de-la-Mata et al., 2015).

\section{Conclusiones}

Se concluye que ambos tratamientos J-Synch más gonadotropina sérica de yegua preñada (eCG) a las $60 \mathrm{~h}$ y $72 \mathrm{~h}$, no mostraron diferencias significativas para el desarrollo folicular al momento del retiro del dispositivo para la inseminación artificial a tiempo fijo (IATF) y en el tamaño del cuerpo lúteo medido al día siete posterior a la misma. Sin embargo, la tasa de preñez fue superior para el protocolo de $60 \mathrm{~h}$. 


\section{Literatura citada}

Baruselli, P.S., G.A. Bó, E.L. Rais, O. Marques, e E.F. Sá-Filho. 2005. Introducaosa IATF no manejo reproductivo de rebanhos bovinos de corte no Brasil. Em: M. Caccia, editor, $6^{\circ}$ Simposio Internacional de Reproducción Animal. Instituto de Reproducción Animal Córdoba, Córdoba, ARG. p. 151-176.

Bernardi, S., R. Allende, R. Mazeo, J. Monti, y P.R. Marini. 2011. Evaluación de los cambios ocasionados en espermatozoides bovinos por variaciones en el manejo de las dosis durante su manipulación en inseminación artificial. InVet. 13(2):25-39.

Ben, G.A., O.E. Goitia, I.F. Mujica, C.J. Munar, y A.M. Valdez. 2002. Programa de inseminación artificial a tiempo fijo. Manual de procedimientos. Sitio rargentino de reproducción animal, ARG. http://www.produccion-animal.com.ar/informacion_ tecnica/inseminacion_artificial/15-programa_inseminacion_a_tiempo_fijo_.pdf. (consultado 20 jun. 2017).

Bó, G.A., y P.S. Baruselli. 2002. Programas de inseminación artificial a tiempo fijo en el ganado bovino en regiones subtropicales y tropicales. En: C. González-Stagnaro et al., editores, Avances en la ganadería doble propósito. Fundación Girarz, Maracaibo, VEN. p. 499-514.

Bó, G.A., L. Cutaia, G.M. Brogliatti, M. Medina, R. Tríbulo, y H. Tríbulo. 2001. Programas de inseminación artificial a tiempo fijo en ganado bovino utilizando progestágenos y estradiol. En: M. Caccia, editor, Resúmenes, Cuarto Simposio Internacional de Reproducción Animal. Instituto de Reproducción Animal Córdoba,Córdoba, ARG. p. 117-136.

Bridges, G.A., J.K. Ahola, C. Brauner, L.H. Cruppe, J.C. Currin, M.L. Day, P.J. Gunn, J.R. Jaeger, S.L. Lake, G.C. Lamb, G.H. Marquezini, R.K. Peel, A.E. Radunz, J.S. Stevenson, and W.D. Whittier. 2012. Determination of the appropriate delivery of prostaglandin F2 $\alpha$ in the five-day CO-Synch + controlled intravaginal drug release protocol in suckled beef cows. J. Anim. Sci. 90:4814-4822. doi: 10.2527/jas.2011-4880.

Bridges, G.A., L.A. Hesler, D.E. Grum, M.L. Mussard, C.L. Gasser, and M.L. Day. 2008. Decreasing the interval between GnRH and PGF $2 \alpha$ from 7 to 5 days and lengthening proestros increases timed-IA pregnancy rates in beef cow. Theriogenology 69:843-851. doi:10.1016/j.theriogenology.2007.12.011.

Bridges, G.A., M.L. Mussard, C.R. Burke, and M.L. Day. 2010. Influence of the length of proestros on fertility and endocrine function in female cattle. Anim. Reprod. Sci. 117: 208-215. doi:10.16/j.anireprosci.2009.05.002.

Bridges, G.A., M.L. Mussard, L.A. Hesler, and M.L. Day. 2014. Comparison of follicular dynamics and hormone concentrations between the 7-day and 5-day CO-Synch + CIDR program in primiparous beef cows. Theriogenology 81:632-638. doi:10.1016/j.theriogenology.2013.11.020.

Cerri, R.L., H.M. Rutigliano, R.C. Chebel, and J.P. Santos. 2009. Period of dominance of the ovulatory follicle influences embryo quality in lactating dairy cows. Reproduction 137:813-823. doi:10.1530/REP-08-0242.

Cutaia, L. 2011. Uso sistemático de eCG asociado a protocolos de inseminación artificial a tiempo fijo en ganado de carne y leche. Presentado en: I Simposio Latinoamericano de Reproducción Animal, Hotel Casino Enjoy Viña, Viña del Mar, CHI, 7-8 nov. Presentación 10.

Cutaia, L., G. Veneranda, R. Tribulo, P.S. Barucelli, y G.A. Bó. 2003. Programas de inseminación artificial a tiempo fijo: Análisis de factores que afectan los resultados. En: M. Caccia, editor, V Simposio Internacional de Reproducción Animal. Instituto de Reproducción Animal Córdoba, Córdoba, ARG. p. 119.

de-la-Mata, J.J., y G.A. Bó. 2012. Sincronización de celos y ovulación utilizando protocolos de BE y GnRH en periodos reducidos de inserción de un dispositivo con progesterona en vaquillonas para carne. Taurus 55:17-23.

de-la-Mata, J.J., M. Ré, and G.A. Bó. 2015. Combination of estrus detection and fixed-time artificial insemination in beef heifers following shortened estradiol-based protocol that provides for a lengthened proestrus. Reprod. Fert. Dev. 27:9697. doi:10.1071/RDV27n1Ab8 
Guáqueta, H., J. Zambrano, and C. Jiménez. 2014. Risk factors for ovarian postpartum resumption in Holstein cows, under high tropical conditions. Rev. MVZ Córdoba 19:3970-3983.

Hansel, W., and H.W. Alila. 1984. Causes of postpartum anoestrus in cattle in the tropics. In: International Atomic Energy Agency, editor, Nuclear techniques in tropical animal diseases and nutritional disorders. International Atomic Energy Agency, Vienna, AUT. p. 21-40.

Menchaca, A., R. Nuñez-Olivera, F. Cuadro, and G. Bo. 2015. Pregnancy rates in beef heifers synchronized with a shortened oestradiol-based treatment that provides for a prolonged proestrus. Reprod. Fert. Dev. 27:96. doi:10.1071/RDv27n1 Ab7.

Motta-Delgado, P.A., L.G. Rivera-Calderon, A.A. Mariño, and C.E. Lizcano. 2012. Productive and reproductive performance of F1 Gyr x Holstein cows in Colombian warm climate. Vet. Zootec. 6:17-23.

Núñez-Olivera, R., T. de-Castro, C. García-Pintos, G.A. Bó, J. Piaggio, and A. Menchaca. 2014. Ovulatory response and luteal function after eCG administration at the end of a progesterone and estradiol' based treatment in postpartum anestrous beef cattle. Anim. Reprod. Sci. 146:111-116. doi:10.1016/j.anireprosci.2014.02.017.

Perry, G.A., M.F. Smith, A.J. Roberts, M.D. McNeil, and T.W. Geary. 2007. Relationship between size of the ovulatory follicle and pregnancy success in beef heifers. J. Anim. Sci. 85:684-519. doi:10.2527/jas.2006-519

Pitaluga, P.C.S.F., M.F. Sá-Filho, J.N.S. Sales, P.S. Baruselli, and L. Vincenti. 2013. Manipulation of the proestrous by exogenous gonadotropin and estradiol during a timed artificial insemination protocol in suckled Bos indicus beef cows. Liv. Sci. 154:229-234. doi: 10.1016/j.livsci.2013.02.023.

Sartori, R., P.M. Fricke, J.C. Ferreira, O.J. Ginther, and M.C. Wiltbank. 2001. Follicular deviation and acquisition of ovulatory capacity in bovine follicles. Biol. Reprod. 65:1403-1409.

Van-Eerdenburg, F.J., D. Karthaus, M.A. Taverne, I. Mericsy, and O. Szenci. 2002. The relationship between estrus behavioral score and time of ovulation in dairy cattle. J. Dairy Sci. 85:1150-1156. doi:10.3168/jds.S0022-0302(02)74177-5

Yánez, D., I. Barbona, J.C. López, R. Quinteros, S. Bernardi, and P.R. Marini.2016. Possible factors affecting pregnancy rate of cows in the amazon ecuatorian. In: E. Mellisho, editor, Proceedings VI Peruvian Congress Animal Reproduction. SPERMOVA, PER. p. 66. 